\title{
The value of epigenetic biomarkers in breast cancer
}

\author{
Paola Parrella*,1 \\ 'Laboratory of Oncology, IRCCS 'Casa Sollievo della Sofferenza', 71013 San Giovanni Rotondo (FG), Italy \\ *Author for correspondence: Tel.: +39 0882416261; Fax: +39 0882416264; pparrella@operapadrepio.it
}

\section{'Since epigenetic traits are potentially reversible, they represent interesting therapeutic targets."}

First draft submitted: 1 June 2018; Accepted for publication: 21 June 2018; Published online: 25 July 2018

Keywords: biomarkers $\bullet$ breast cancer $\bullet$ diagnosis $\bullet$ DNA methylation $\bullet$ DNMTi $\bullet$ epigenetics $\bullet$ HDACi $\bullet$ histones PMTs • ncRNAs • prognosis

Methylation at cytosines contained within cytosine-phosphate-guanine $(\mathrm{CpG})$ dinucleotides and post-translational modifications (PTMs) of histone proteins tail represent the major components of epigenetic regulation in eukaryotes. In normal conditions, these mechanism finely control cell proliferation and differentiation, thus their deregulation may are deeply involved in cancer development and progression [1]. In the early stages of breast carcinogenesis epigenetic deregulation alter chromatin structure affecting cellular plasticity and supporting the oncogenic reprogramming of cancer progenitor cells with the acquisition of uncontrolled self-renewal abilities [2]. At later stages of cancer development, further epigenetic modifications, together with clonal mutations and signals from the microenvironment, are able to modulate the phenotype of cancer cell and influence their propensity to metastatic spread $[2,3]$. Beyond the classical epigenetic mechanisms, in recent years, noncoding RNAs (ncRNAs), especially miRNAs and lncRNAs have emerged as important epigenetic modifiers [4]. Since epigenetic traits play such central biological roles in breast cancerogenesis, they represent suitable biomarkers for early diagnosis, prognosis or therapy response.

\section{Promoter DNA methylation \& breast cancer diagnosis}

Most of the breast cancer patients diagnosed at early stages of disease show sensational survival rates [5]. Thus, early detection of the disease is the key for a successful management of breast cancer patients. Aberrant DNA methylation often occurs within CpGs island located in the promoter region of tumor suppressor genes contributing to their silencing cancer cells. This modifications are early events in breast cancerogenesis and methylated DNA can be detected with an high degree of specificity in clinical samples. Indeed, promoter hypermethylation was detected with a high concordance in fine needle aspirate and paired primary tumors. Nevertheless, it's detection in clinical samples, did not show a better sensitivity and specificity as compared with routine cytological examination [6]. Several studies have demonstrated that aberrant promoter methylation can be detected also in DNA obtained from a typical and malignant breast cancer cells in ductal lavage or spontaneous produced ductal fluid (Nipple Aspirate). Unfortunately, promoter methylation was also found in nipple aspirate from healthy individuals, and the analysis was unable to distinguish between preinvasive and invasive breast lesions [7].

Several studies have demonstrated the possibility to detect genetic abnormalities in cell-free DNA obtained from plasma (liquid biopsy), and this approach has been brought into the clinic to detect actionable mutations in lung cancer. Similarly, Hoque et al. [8] explored the possibility to detect CpG methylation in cell-free DNA from plasma samples for early cancer detection. They demonstrated that methylation of the selected genes increases during breast cancer progression, and that a panel of possible methylated cancer-specific genes could improve the sensitivity and specificity of this approach. More recently, together with circulating DNA, the recognized stability of ncRNAs, especially miRNAs, in bodily fluids has unveiled the capabilities of circulating ncRNAs to help diagnose breast cancer [9]. 


\section{Epigenetic traits may refine the molecular classification of breast cancer}

Despite the encouraging survival rates in early breast cancer, around $10 \%$ of patients experience tumor relapse [5]. In the attempt to improve our abilities to identify breast cancer patients at higher risk for disease progression, combined gene-expression and DNA copy number analyses, identified five biologically and clinically relevant intrinsic breast cancer subtypes [10]. The two ER positive subtypes show respectively, relatively low (luminal A) and high (luminal B) expression of proliferation-related genes. The HER2-enriched subtype is characterized by the absence of ER expression and presence HER2-amplification/overexpression. The basal-like subtype shows absence of ER, PgR expression and HER2 amplification, whereas the normal-like subtype shows a similar expression profile to normal breast tissue [10]. These molecular subgroups correlate with patient's survival and several gene expression based risk scores have been developed to identify low risk breast cancer patients in the attempt to spare unnecessary adjuvant treatment. However, even this classification system cannot account for all the reported pathological and clinical heterogeneity of breast tumors. Several studies have investigated the potentials of DNA methylation profiling and core histone modifications, to better refine the molecular classification and improve the clinical management of breast cancer patients. In a pioneering study, Holm et al. [11] found that luminal A, luminal B and basal-like, breast cancer subtypes show specific methylation profiles. Successively, a number of studies have combined DNA methylation profiling with gene expression analyses to identify molecular signatures that may better account for the pathological and clinical heterogeneity of breast tumors [12]. While luminal A and HER2 enriched subtypes do not show specific methylation patterns, these studies agree in identifying specific methylation cluster (epitypes) for basal-like and luminal B breast tumors [13].

The possibility to use histone PTMs as classifiers has been evaluated in breast cancer by using immunohistochemistry. Elsheikh et al. [14] analysed histones 3 and 4 PTMs on a large series of breast cancer cases. Relatively high levels of global histone acetylation and methylation, were found in the luminal-like subtype. Whereas, HER2 amplified and triple negative/basal-like subtypes, showed moderate to low levels of lysine acetylation, lysine and arginine methylation. In particular, loss of histone $\mathrm{H} 4$ lysine 20 trimethylation was associated with luminal subtypes and it was independently associated with poor prognosis in multivariable analysis [14].

\section{DNA methylation \& response to breast cancer treatments}

In recent years, advances in translational research have allowed the incorporation of more specific agents in breast cancer standard therapies. In line with the ambition of precision medicine, understanding the epigenetic mechanisms underlying cancer behavior holds great potential for optimizing the therapeutic interventions and thus identifying individuals who might benefit from improved modalities and treatment strategies. Several studies demonstrated that DNA methylation in cancer-associated genes correlates with resistance to endocrine therapies, chemotherapies and anti-HER2 treatments [1]. In particular the aberrant methylation of PITX2 promoter was associated with poor prognosis in ER positive, lymph node negative, patients treated only with hormone therapy [15]. Adding a taxane to chemotherapy improves the outcome of advanced and high risk breast cancer patients. In a small patients cohort, we have found that aberrant promoter methylation of the KEAP1 gene, a central negative regulator of the cell adaptive response to oxidative stress, was associated with a better overall survival in patients' receiving sequential therapy with anthracyclines and cyclophosphamide followed by taxanes [16]. Thus our data suggest that KEAP1 methylation might confer resistance to chemotherapy regimens including taxanes. Recently PARP1 inhibitors have entered into clinical practice for the treatment of BRCA mutated ovarian and breast cancers. Interestingly, in an in vitro study $B R C A 1$ gene hypermethylation was able to confer the same degree of sensitivity to three different PARP inhibitors as the presence of $B R C A 1$ mutations, suggesting that this epigenetic abnormality could represent a hallmark of BRCAness [17]. However, current clinical data do not support a role of promoter methylation in the response to PARP1 inhibitors in BRCA1 wild-type tumors.

\section{Epigenetic therapies in breast cancer}

Since epigenetic traits are potentially reversible, they represent interesting therapeutic targets. Therefore, the antitumor activity of DNA methyl transferases inhibitors and histone deacethylatese inhibitors (HDACi), has been evaluated in several tumor types including breast cancer. 5-azacytidine (zacytidine), 5-aza-2'-deoxycytidine (decitabine) and hydralazine inhibit DNMTs by forming a covalent bond with the enzyme after incorporation into DNA. HDACi enhance the acetylation of cellular proteins by blocking the HDAC activity. While these drugs have been approved by drug regulatory agencies for the clinical use in hematological malignancies, their efficacy is still under investigation on solid tumor [18]. In in vitro studies both DNA methyl transferases inhibitors and 
HDACi have demonstrated antitumor activity in breast cancer cell lines. However, many of these compounds, did not show clinical benefit in Phase I and II clinical trials [1]. The most promising results have been obtained with the HDAC inhibitor Entinostat, which potently and selectively inhibits class I and IV HDAC enzymes. In preclinical studies Entinostat, was able to reverse the EMT phenotype in breast cancer cell lines [19]. In a Phase II study the combination Entinost and Examestane showed an overall survival advantage (ENCORE301 trial). An ECOG-ACRIN Phase III registration study is currently ongoing to confirm the efficacy of the combinatory treatment (E2112, NCT02115282) [20].

\section{Conclusion}

There is now a compelling body of evidence supporting a possible role of epigenetic traits as molecular biomarkers in breast cancer. However, a global review of the current literatures, reveals several discrepancies that so far prevent the transfer of this knowledge to patient' bedside. Indeed, existing studies are limited by the retrospective design, different cohorts' size, sample preparation, availability of profiling technologies and data analysis. Therefore, it is necessary to carry on rigorous validation studies on large prospective cohorts to identify epigenetic traits that do show reliability and applicability, as already done in gliomas for $M G M T$ methylation analysis. If this approach will be successful, we might expect that along with the analysis of mRNA expression signatures, cancer-related genes mutations and chromosomal aberrations, the characterization of epigenetic traits will enter the routine clinical investigation with the aim to improve the overall management and monitoring of breast cancer patients.

\section{Acknowledgements}

Work in P Parrella lab is supported by the Italian Ministry of Health (MoH) co-funded by the European Regional Development Fund 'A way of making Europe' under the TRANSCAN ERA-NET on Translational Cancer Research (grant number RRC-2014-2354565 and CANCER13-FP-011); Italian Ministry of Health (MoH) 'Ricerca Corrente 2016' and ' $5 \times 1000$ ' voluntary contributions and 'Associazione Italiana Ricerca sul Cancro' (AIRC) IG-1269/2006.

\section{Financial \& competing interests disclosure}

The authors have no relevant affiliations or financial involvement with any organization or entity with a financial interest in or financial conflict with the subject matter or materials discussed in the manuscript. This includes employment, consultancies, honoraria, stock ownership or options, expert testimony, grants or patents received or pending, or royalties.

No writing assistance was utilized in the production of this manuscript.

\section{References}

1 Pasculli B, Barbano R, Parrella P. Epigenetics of breast cancer: biology and clinical implication in the era of precision medicine. Semin. Cancer Biol. 51, 22-35 (2018).

2 El Helou R, Wicinski J, Guille A et al. Brief reports: a distinct DNA methylation signature defines breast cancer stem cells and predicts cancer outcome. Stem Cells 32(11), 3031-3036 2014.

3 Pal B, Bouras T, Shi W et al. Global changes in the mammary epigenome are induced by hormonal cues and coordinated by Ezh2. Cell Rep. 3(2) 411-426 (2013).

4 Peschansky VJ, Wahlestedt C. Non-coding RNAs as direct and indirect modulators of epigenetic regulation. Epigenetics 9(1), 3-12 (2014).

5 Siegel RL, Miller KD, Jemal A. Cancer statistics, 2018. CA Cancer J. Clin. 68(1), 7-30 (2018).

6 Jerónimo C, Costa I, Martins MC et al. Detection of gene promoter hypermethylation in fine needle washings from breast lesions. Clin. Cancer Res. 9(9) 3413-3417 (2003).

7 Evron E, Dooley WC, Umbricht CB et al. Detection of breast cancer cells in ductal lavage fluid by methylation-specific PCR. Lancet 357(9265) 1335-1336 (2001)

8 Hoque MO, Feng Q, Toure P et al. Detection of aberrant methylation of four genes in plasma DNA for the detection of breast cancer. J. Clin. Oncol. 24(26) 4262-4269 (2006).

9 Schwarzenbach H, Nishida N, Calin GA et al. Clinical relevance of circulating cell-free microRNAs in cancer. Nat. Rev. Clin. Oncol. 11(3) 145-156 (2014).

10 Perou CM, Sørlie T, Eisen MB et al. Molecular portraits of human breast tumors. Nature 406(6797), 747-752 (2000).

11 Holm K, Hegardt C, Staaf J et al. Molecular subtypes of breast cancer are associated with characteristic DNA methylation patterns. Breast Cancer Res. 12(3) R36 (2010). 
12 Stefansson OA, Moran S, Gomez A et al. A DNA methylation-based definition of biologically distinct breast cancer subtypes. Mol. Oncol. 9(3) 555-568 (2015).

13 Gao Y, Jones A, Fasching PA et al. The integrative epigenomic-transcriptomic landscape of ER positive breast cancer. Clin. Epigenetics 7 , 126 (2015).

14 Elsheikh SE, Green AR, Rakha EA et al. Global histone modifications in breast cancer correlate with tumor phenotypes, prognostic factors, and patient outcome. Cancer Res. 69(9) 3802-3809 (2009).

15 Harbeck N, Nimmrich I, Hartmann A et al. Multicenter study using paraffin-embedded tumor tissue testing PITX2 DNA methylation as a marker for outcome prediction in tamoxifen-treated, node-negative breast cancer patients. J. Clin. Oncol. 26(31) 5036-5042 (2008).

16 Barbano R, Muscarella LA, Pasculli B et al. Aberrant Keap1 methylation in breast cancer and association with clinicopathological features. Epigenetics 8(1), 105-112 (2013).

17 Veeck J, Ropero S, Setien F et al. BRCA1 CpG island hypermethylation predicts sensitivity to poly(adenosine diphosphate)-ribose polymerase inhibitors. J. Clin. Oncol. 28(29) e563-e564 (2010)

18 Ronnekleiv-Kelly SM, Sharma A, Ahuja N. Epigenetic therapy and chemosensitization in solid malignancy. Cancer Treat Rev. 55, 200-208 (2017).

19 Schech A, Kazi A, Yu S et al. Histone deacetylase inhibitor entinostat inhibits tumor-initiating cells in triple-negative breast cancer cells. Mol. Cancer Ther. 14(8) 1848-1857 (2015).

20 Connolly RM, Li H, Jankowitz RC et al. Combination epigenetic therapy in advanced breast cancer with 5-azacitidine and entinostat: a Phase II National Cancer Institute/stand up to cancer study. Clin. Cancer Res. 23(11) 2691-2701 (2017). 